\title{
El crecimiento urbano en Europa: conceptos, tendencias y marco comparativo para el área metropolitana de Santiago de Chile ${ }^{1}$
}

\section{European urban growth: concepts, trends and a comparative framework for the metropolitan area of Santiago de Chile}

\author{
Rodrigo Hidalgo* y Axel Borsdorf**
}

Desde el comienzo del período colonial, Latinoamérica ha sido un continente marcado por la fundación de ciudades. El enviado de la corona española, el Encomendero (propietario de la tierra) tenía su residencia principal en la ciudad, donde tomaba lugar la vida económica, social y política.

Las áreas rurales de Latinoamérica son un espacio complementario; las ciudades y sobretodo las grandes aglomeraciones son los centros indiscutibles de control. A pesar de esto, todavía persiste en muchos latinoamericanos el anhelo del modo de vida rural. El culto al gaucho en Argentina o el huaso en Chile son indicadores de ello. Sin embargo, esta afición tiene como regla el disfrute del campo solo en el tiempo libre o en el fin de semana. De este modo, se construyen Country-Clubs en lugar de las tradicionales medialunas para el rodeo, surgiendo un nuevo tejido rural. El "campo" es parte de la identidad, pero se vive en la ciudad. A pesar de que el éxodo desde las zonas rurales ha perdido fuerza (Borsdorf 2004, Parnreiter 2004), muchos habitantes de estas áreas añoran una vida en la ciudad: la libertad, la educación, el trabajo,

\footnotetext{
* Instituto de Geografía [hidalgo@geo.puc.cl]

* * Universidad de Insbruck

${ }^{1}$ Proyectos Fondecyt 1030472 y 1060759 y de Cooperación Internacional 7080114.
} 
comunicación y la promesa del disfrute del tiempo libre. Así crecen las ciudades latinoamericanas, el crecimiento poblacional es menos intenso de lo que era hace dos o tres décadas atrás, pero la superficie urbana crece significativamente y lo hace a costa del suelo rural.

Por su parte, en Europa, al parecer decae el poder de la ciudad. Las grandes ciudades perdieron sus habitantes, muchas se deprimieron por la transformaciones por el cambio del modelo de desarrollo económico. Se han establecido "cinturones de riqueza" en la periferia de las aglomeraciones. El fenómeno de las "ciudades encogidas" no es desde hace mucho tiempo restringido solamente a la Alemania del Este, viviendas vacías se pueden observar también en centros pequeños y medianos de otras regiones europeas, mientras que la construcción de viviendas en la antigua periferia rural de las ciudades todavía esta en auge. Además se produce un nuevo fenómeno: cada vez más funciones centrales (comercio en gran escala y minorista, servicios e instituciones de enseñanza) se establecen en la zona periférica. Las periferias de las ciudades que hasta hace unos 20 años eran zonas suburbanas, es decir un complemento de los suburbios, ahora son entidades diferenciadas funcionalmente, que tienen su propia centralidad.

Los procesos aquí sugeridos son relativamente nuevos, se iniciaron solo a fines de la década de 1970 y desde entonces se han fortalecido. Paralelamente con el avance del neoliberalismo como doctrina económica, la globalización ha tenido una importante influencia en los procesos. Las ciudades, al parecer, son el "mundo en transición" (Schneider-Sliwa 2002). Esto plantea la cuestión de por qué un factor desencadenante puede provocar resultados diferentes.

El debate europeo sobre la destitución de las antiguas formas de desarrollo suburbano, a través de estructuras completamente nuevas junto al retroceso de las diferencias entre ciudad y campo, comenzó con el pensamiento de T. Sieverts con la formulación del concepto de "fenómeno del interurbano" en 1997. Numerosos autores han abordado esta problemática y aportado nuevos términos (Postsuburbia: Borsdorf 2004; Stadtland: Eisinger \& Schneider 2003; Ville emergente: Piron \& Dubois-Taine 1998, entre otros) o realizaron estudios comparativos en espacios europeos (Burdack, Herfert \& Ruldoph 2005; Dubois-Taine 2004).

Las constataciones que se exponen en este artículo se basan, principalmente, en los resultados de proyectos de la Unión Europea (COMET, COST C10) en la que uno de los autores estuvo involucrado. Debido al planteamiento eurocentrista aplicado muchas veces a tabla rasa, la pregunta sobre la diferencia de desarrollo entre Europa y Latinoamérica ha quedado sin resolver y es parte de los objetivos de este documento. 
El trabajo se adentra en el análisis e interpretación del crecimiento urbano reciente de las ciudades europeas e indaga en la aplicación y proyección de estos cambios en la realidad de América Latina, teniendo como especial referencia lo ocurrido en el área metropolitana de Santiago de Chile.

\section{TERMINOLOGÍA}

Los siguientes constataciones han sido reportadas en muchas partes del mundo. Ellos son, por lo tanto, resultados probables de la globalización y por esto se interpretan como alineaciones globales de procesos espaciales. Pero existen diferencias, incluso en la terminología asociada. En Norteamérica, el fenómeno del surgimiento de las edge cities y más allá aún, de tendencias de exurbanización, desurbanización y contraurbanización, son en cierta manera comparables, pero difieren sustancialmente de los desarrollos en Europa y América Latina.

El término inglés edge city fue propuesto por Garreau en 1991. Caracteriza a grandes centros en las afueras de las ciudades y que son multifuncionales, los cuales contrastan con la Post-Suburbia, sobre todo en las características distintivas que hacen de esta última ser una ciudad independiente. La Edge city se caracteriza de la siguiente manera: tiene sobre $450.000 \mathrm{~m}^{2}$ de superficie para oficinas y más de $55.000 \mathrm{~m}^{2}$ de superficie para el comercio minorista. A menudo su superficie de oficinas es más grande que en la ciudad tradicional. Ellas se encuentran por regla general a grandes distancias de la ciudad tradicional (hasta $50 \mathrm{Km}$.). Por ello tiene la edge city un especial significado en el ámbito de las migraciones pendulares y un balance positivo en este proceso. Del mismo modo, la Post-Suburbia se expande en superficie de la edge city, que antes se utilizaba con fines agrícolas.

Las periféricas edge city no son ciudades políticamente independientes. Ellas son de alguna manera evaluadas como la etapa final del proceso de posturbanización. Ellas son en Estados Unidos, a diferencia del desarrollo post-suburbano en Europa, asociadas con grandes desventajas para las ciudades tradicionales, cuyos centros se degradan y sus terrenos baldíos en zonas comerciales son altamente apetecidos.

En los Estados Unidos es también reconocida una fuerte tendencia a la exurbanización. Se entiende como un rasgo fuerte de la ocupación residencial de baja densidad y de otros usos terciarios en las zonas rurales, lo que da a lugar espacios estructurados con claridad. Estas personas conservan sus puestos de trabajo en el centro de las ciudades. De un modo diferente se comporta la 
desurbanización. Constituye una forma especial del proceso de contracción de las ciudades, que también se ha observado en Europa, particularmente en los nuevos Estados Federales de Alemania. Estos movimientos emigratorios no solo son de población, sino que también de puestos de trabajo, los cuales son creados en otros lugares con mejores condiciones -ventajas competitivas-, provocando que las localizaciones originales sean abandonadas. Este proceso puede intensificar el proceso de post-urbanización. La contraurbanización es otro caso especial de desurbanización. Debido al mejoramiento de la accesibilidad a los espacios rurales y la mejor calidad de vida existente en ellos, se producen movimientos migratorios de población y empresas a ciudades pequeñas o medianas o bien a zonas rurales. Finalmente, se debe mencionar el fenómeno de la "migración por amenidades", reconocida por primera vez en los Estados Unidos, pero que hoy se puede encontrar en muchas partes del mundo, incluso en Europa y América Latina. Siguiendo a Moss (2006) este es el tipo de residencia de población urbana que se ha trasladado a áreas con altos estándares de calidad de vida y paisaje escénico; para Moss este tipo de migración se produce solo en los ambientes de montaña. En estas zonas algunos migrantes conservan sus puestos de trabajo y se transforman en "migrantes interurbanos", que se sirven de las nuevas tecnologías de la información para seguir desde su nuevo lugar de residencia su trabajo en empresas, las cuales se encuentran aún en las grandes aglomeraciones. Ellos son "migrantes de fin de semana" que por tres a cuatro días disfrutan las "amenidades" de una nueva (segunda) residencia o buscan allí su lugar de descanso una vez que han jubilado.

En Europa, en parte erróneamente, los conceptos de periurbanización y suburbanización han sido utilizados como sinónimos de post-urbanización. La suburbanización es claramente una etapa anterior a la post-suburbanización. Los barrios suburbanos se encuentran también cercanos al centro de la ciudad, ellos se caracterizan también porque ostentan un alto nivel de población en las noches y una escasa cantidad de ella en el día. Además son equipados funcionalmente solo de manera básica y por lo tanto corresponden a espacios complementarios para el centro de la ciudad. Ellos representan el antiguo modelo gravitacional de ciudad, con un centro compacto, demográfica y funcionalmente potente y luego una clara frontera divisoria.

El concepto de periurbanización se origina en Francia y hoy se emplea también en Suiza. En la literatura de lengua germana tiene menos fuerza y posee muchas definiciones equívocas. En ocasiones se denomina periurbanización también como rururbanización. Longchamp (1989) entiende como comunas periurbanas (communes périurbaines) no solo a edificaciones resi- 
denciales anexas, sino que a la penetración de áreas urbanas residenciales en el medio rural. Más del $40 \%$ de la población activa migra a una aglomeración o su área de influencia más cercana. A diferencia de la suburbanización las áreas de expansión a gran distancia de la ciudad se agrupan en torno a los antiguos núcleos rurales; a causa de la gran distancia de la ciudad, es también una localización adecuada para el establecimiento de lugares de abastecimiento (tales como supermercados).

En la siguiente matriz de características se presentan los diferentes contrastes que poseen los términos señalados (tabla 1).

TABLA 1.

MATRIZ DE TERMINOLOGÍA DEL CRECIMIENTO URBANO

\begin{tabular}{|c|c|c|c|c|c|c|c|}
\hline & Suburbia & Periurbia & $\begin{array}{l}\text { Post- } \\
\text { Suburbia }\end{array}$ & Exurbia & $\begin{array}{c}\text { Counter- } \\
\text { urbia }\end{array}$ & $\begin{array}{l}\text { Desurba- } \\
\text { nización }\end{array}$ & $\begin{array}{l}\text { Edge } \\
\text { City }\end{array}$ \\
\hline $\begin{array}{l}\text { Relación con el } \\
\text { núcleo tradicional } \\
\text { de la ciudad }\end{array}$ & Fuerte & $\begin{array}{l}\text { Todavía } \\
\text { fuerte }\end{array}$ & Escasa & Fuerte & $\begin{array}{l}\text { Todavía } \\
\text { fuerte }\end{array}$ & Escasa & $\begin{array}{l}\text { Muy } \\
\text { escasa }\end{array}$ \\
\hline $\begin{array}{l}\text { Distancia al núcleo } \\
\text { tradicional } \\
\text { de la ciudad }\end{array}$ & Escasa & Media & Media & $\begin{array}{l}\text { Media a } \\
\text { grande }\end{array}$ & $\begin{array}{l}\text { Media a } \\
\text { grande }\end{array}$ & Grande & Grande \\
\hline Migración pendular & $\begin{array}{l}\text { Emigración } \\
\text { diaria }\end{array}$ & $\begin{array}{l}\text { Emigración } \\
\text { diaria }\end{array}$ & $\begin{array}{c}\text { En } \\
\text { equilibrio }\end{array}$ & $\begin{array}{c}\text { Emigración } \\
\text { diaria }\end{array}$ & $\begin{array}{l}\text { Emigración } \\
\text { diaria }\end{array}$ & No hay & $\begin{array}{c}\text { Inmigración } \\
\text { diaria }\end{array}$ \\
\hline Centralidad & No hay & No hay & $\begin{array}{c}\text { Desde } \\
\text { funciones } \\
\text { individuales } \\
\text { a medias } \\
\text { y altas }\end{array}$ & No hay & No hay & - & Alta \\
\hline $\begin{array}{l}\text { Pérdida para el } \\
\text { núcleo urbano }\end{array}$ & No hay & Población & $\begin{array}{l}\text { Población } \\
\text { y puestos } \\
\text { de trabajo }\end{array}$ & Población & $\begin{array}{l}\text { Población } \\
\text { y puestos } \\
\text { de trabajo }\end{array}$ & Población & $\begin{array}{l}\text { Población } \\
\text { y puestos } \\
\text { de trabajo }\end{array}$ \\
\hline $\begin{array}{l}\text { Fuerza motivadora } \\
\text { de la movilidad }\end{array}$ & $\begin{array}{c}\text { Viviendas } \\
\text { baratas }\end{array}$ & $\begin{array}{c}\text { Viviendas } \\
\text { baratas }\end{array}$ & $\begin{array}{l}\text { Estilo y } \\
\text { calidad } \\
\text { de vida }\end{array}$ & $\begin{array}{l}\text { Calidad } \\
\text { de vida }\end{array}$ & $\begin{array}{l}\text { Asequibilidad } \\
\text { y calidad } \\
\text { de vida }\end{array}$ & $\begin{array}{c}\text { Ventajas } \\
\text { competitivas } \\
\text { para } \\
\text { empresas }\end{array}$ & $\begin{array}{c}\text { Ventajas } \\
\text { competitivas } \\
\text { para } \\
\text { empresas }\end{array}$ \\
\hline
\end{tabular}

La figura 1 se esquematiza el desarrollo de la ciudad europea. Hasta mediados del siglo XIX, la dicotomía campo-ciudad era absolutamente clara, tanto a 
nivel económico, social, cultural y jurídico. Sin embargo, con los cambios sucedidos en el siglo decimonónico, comienza a constituirse un continuo campo-ciudad (suburbanización). En las últimas décadas del siglo xx, es posible apreciar una complejización de la estructura espacial de la ciudad, adquiriendo una forma de un archipiélago urbano. En la figura, las diferencias entre suburbio, periurbano y post-suburbia se encuentran representadas a través de la intensidad de las flechas (i.e. mano de obra, poder adquisitivo).

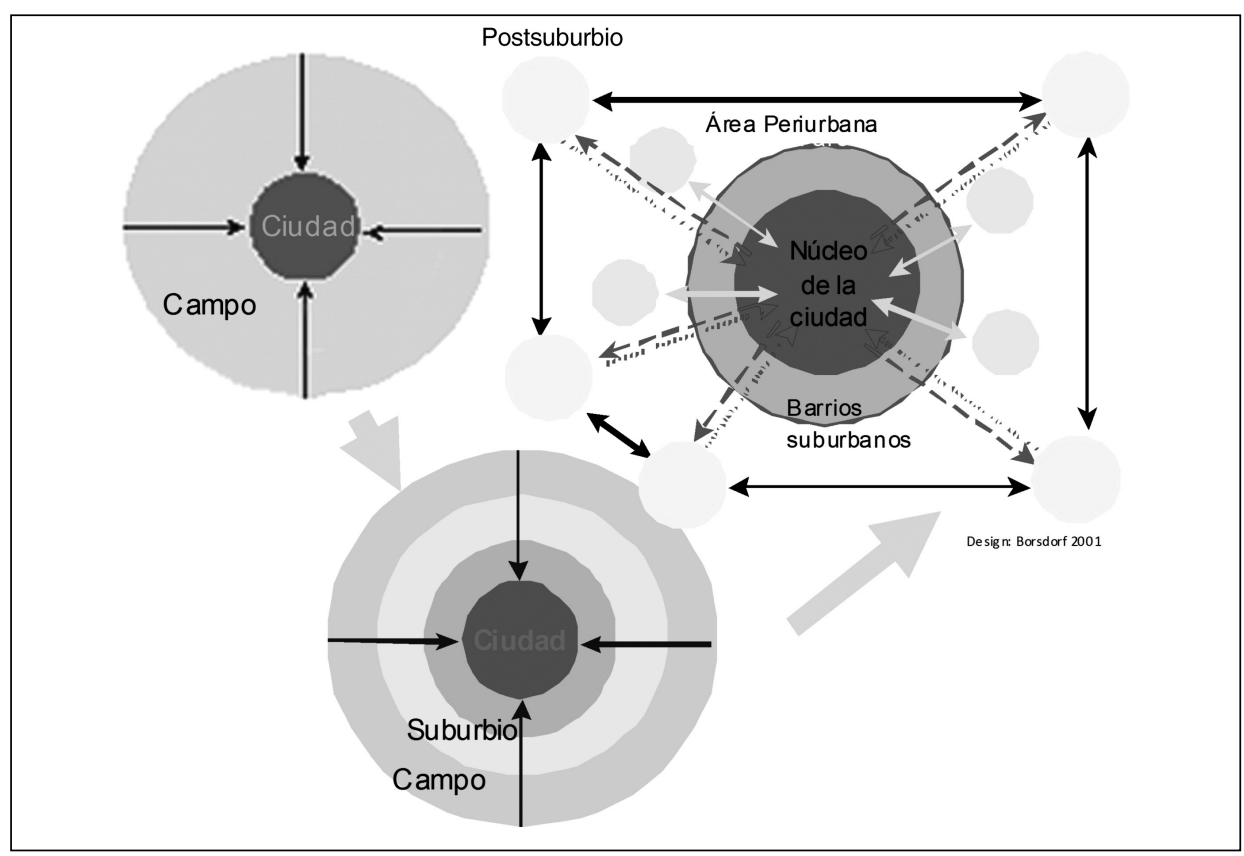

Fuente: Elaboración propia.

Figura 1. Modelo de desarrollo de las ciudades en Europa desde la dicotomía urbano-rural a la interconexión urbana-rural

El DESARROLlO URBANO EN EUROPA

La urbanización en Europa, es ante todo una consecuencia del crecimiento de la población en el siglo xIx, la cual fue favorecida por las nuevas libertades que ofrecía la ciudad (liberación del campesino y liberalización del comercio), 
la industrialización, las mejoras públicas constantes de las infraestructuras de transporte y el surgimiento de nuevas estructuras en la industria de la construcción. En cerca de 150 años se han considerado los mismos hechos detonantes como causas del crecimiento de las ciudades: crecimiento económico, cambio tecnológico, el aumento de la demanda de viviendas, disminución de los costos de transporte y los ideales nostálgicos del modo de vida, representados en la "green-haus", además de la búsqueda de la propiedad individual, suelo y vivienda (Borsdorf, 2002).

Desde aproximadamente 1980 la urbanización en Europa adquirió una nueva cualidad, que puede ser observada prácticamente en todas partes. A este fenómeno fue dedicada una acción aplicada por el programa COST (C10), a través de un proyecto FP5 de la Unión Europea (COMET: www.comet.ac.at), que ha examinado otros aspectos de la "nueva" urbanización (ver en particular Burdack, Herfert \& Rudolph 2005; Brake, Dangschat \& Herfert 2001). Este estudio ha constatado que los clásicos elementos detonantes de la urbanización se acercan hoy a nuevas fuerzas: el aumento del bienestar, la desregularización de la planificación urbana, nuevos actores en el mercado inmobiliario, las tendencias centrífugas de los comercios minoristas, el aumento de la prestación de servicios (empresas de servicio, logística, capacitación), nuevas tecnologías de la información y las comunicaciones (ICT), las nuevas formas de trabajo móviles independientes del lugar, así como la creciente competencia entre las municipalidades para atraer empresas y nuevos residentes.

Los resultados anteriormente señalados pueden ser descritos de manera más certera con el término "post-suburbanización" (Burdack 2001; Borsdorf 2005). Luego de la fase de suburbanización, en la cual la periferia urbana poseía ciudades dormitorio monofuncionales, a menudo hoy se desarrollan en el entorno urbano, múltiples pequeños centros polifuncionales, que frecuentemente, pero no siempre, se agrupan alrededor de una función principal. Actividades de esta índole son los centros comerciales, centros de entretenimiento, parques de negocios, universidades (privadas), aglomeraciones de mercados especializados no integrados, pero también alrededor de canchas de golf, sky y country clubs, tal como se observa en la figura 2.

El desarrollo sigue los antiguos modelos del desarrollo urbano (en el modelo se reconoce la concepción punto-axial de la ciudad regional), los cuales son parcialmente re-interpretados y profundizados, pero también cuestionados o abandonados. La Post-suburbia se desarrolla más y más hacía la estructura dominante de la ciudad postmoderna, con esto se desvanecen pro- 


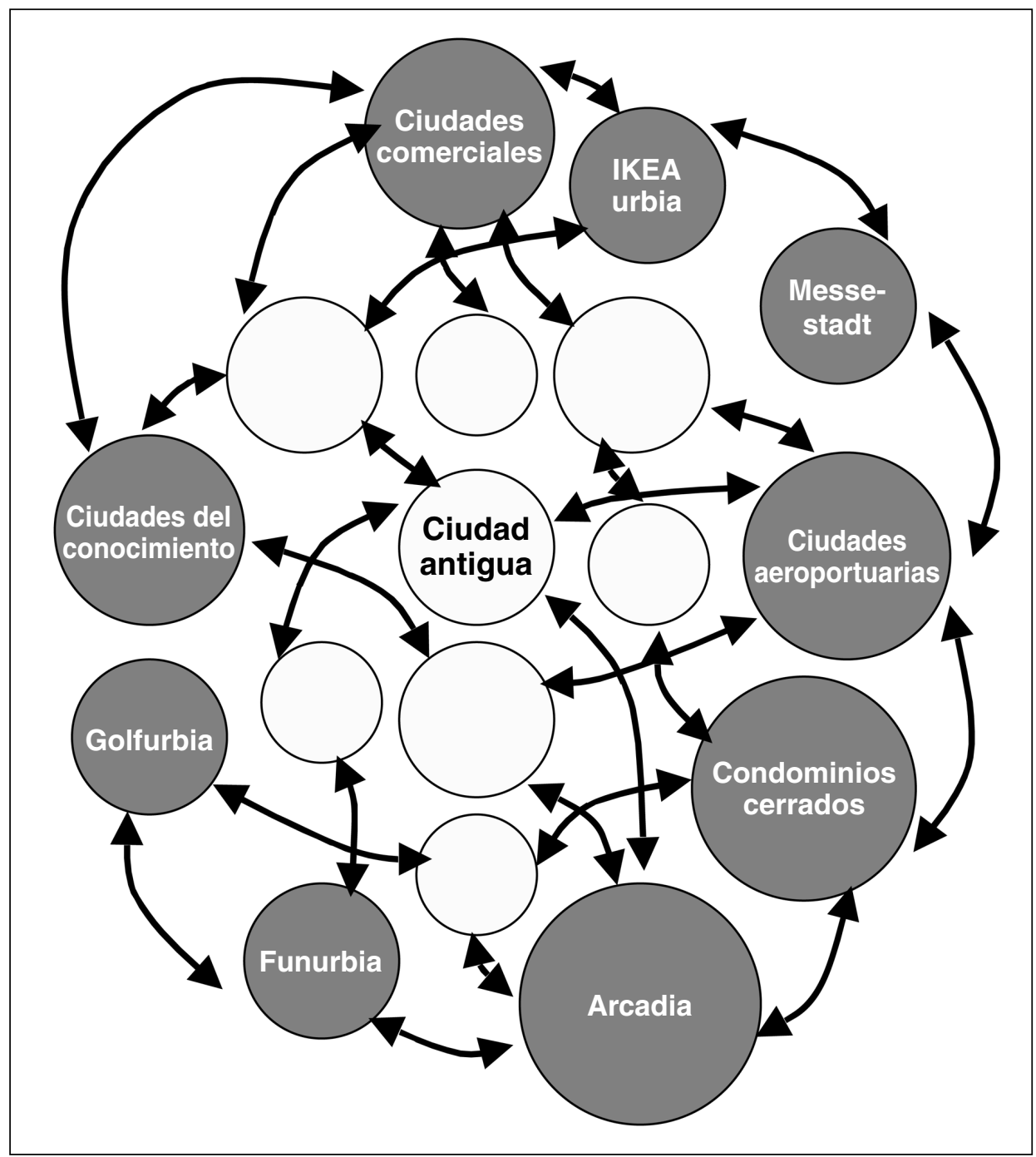

FIgURA 2. Modelo de la aglomeración Post-Suburbana. Fuente: Borsdorf, 2004

gresivamente los antiguos patrones y modelos (centralidad, gravitación, distancia, ordenamiento, racionalidad).

Una comparación de los antiguos y nuevos conceptos urbanos en Europa ilustra el proceso (tabla 2): 
TABLA 2

LA CONCEPCIÓN DE CIUDAD MODERNA Y POSTMODERNA, UNA COMPARACIÓN

\begin{tabular}{|c|c|c|}
\hline & Ciudad "moderna" & Ciudad "postmoderna" \\
\hline \multirow[t]{9}{*}{ Contenido } & Objeto primario: Forma & Objeto primario: Función \\
\hline & $\begin{array}{l}\text { Pregunta: ¿cuáles estructuras? } \\
\text { ¿Dónde? }\end{array}$ & $\begin{array}{l}\text { Pregunta: ¿cuáles estructuras? } \\
\text { ¿Dónde? }\end{array}$ \\
\hline & Estático & Dinámico \\
\hline & Estado actual & Antes-ahora-después \\
\hline & Emplazamiento & Preferencia de emplazamiento \\
\hline & $\begin{array}{l}\text { Todas las actividades } \\
\text { económicas }\end{array}$ & $\begin{array}{l}\text { Tecnologías de información y } \\
\text { "nueva" economía }\end{array}$ \\
\hline & Ciudad compacta & $\begin{array}{l}\text { Corredores, clústers, cintas, } \\
\text { clones }\end{array}$ \\
\hline & Espacios funcionales & $\begin{array}{l}\text { Espacios de acción, Espacios } \\
\text { de resultados }\end{array}$ \\
\hline & Explotación monofuncional & Mezcla de funciones \\
\hline \multirow[t]{7}{*}{ Meta-Idea } & Gran línea & Voluntarismo \\
\hline & Gran idea & Variedad de ideas \\
\hline & Drama & Comedia \\
\hline & $\begin{array}{l}\text { Grandes Teorías (por ejemplo } \\
\text { centralidad }\end{array}$ & Deconstrucción \\
\hline & Jerarquía & Mosaico, Patchwork \\
\hline & Estructuras rígidas & Estructuras flexibles \\
\hline & Información sobria & Discurso, Polémica \\
\hline \multirow[t]{4}{*}{ Meta } & Gobierno top-down & Gobernanza, bottom-up \\
\hline & Una perspectiva & Muchas perspectivas \\
\hline & Apariencia objetiva & Pérdida de objetividad \\
\hline & Imagen & Logo \\
\hline \multirow[t]{4}{*}{ Relaciones Públicas } & Síntesis & Análisis y síntesis \\
\hline & Bidimensional & Tri y cuatridimensional \\
\hline & Impresas & En Web e interactivas \\
\hline & Difusión bajo especialistas & Difusión abierta \\
\hline
\end{tabular}


El desarrollo en Santiago de Chile

La urbanización en el subcontinente latinoamericano muestra una evolución de sus cambios más rápidos de los que se han vivido en Europa en un período más corto de tiempo y, por lo mismo, con expresiones morfológicas aparentemente similares, pero que encierran velocidades y manifestaciones diferentes.

La figura 3 muestra esquemas estructurales generalizados de la ciudad en América Latina, situados siempre al final de importantes fases de la urbanización, como son la época colonial (1820), la primera fase de urbanización influida fuertemente por la inmigración europea (1920), la segunda fase de urbanización marcada por el éxodo rural y la migración interna (1970) y la ciudad contemporánea (2000). En estas etapas, la ciudad cambió desde un cuerpo muy compacto a un perímetro sectorial, desde un organismo polarizado a una ciudad fragmentada (Borsdorf, 2003).

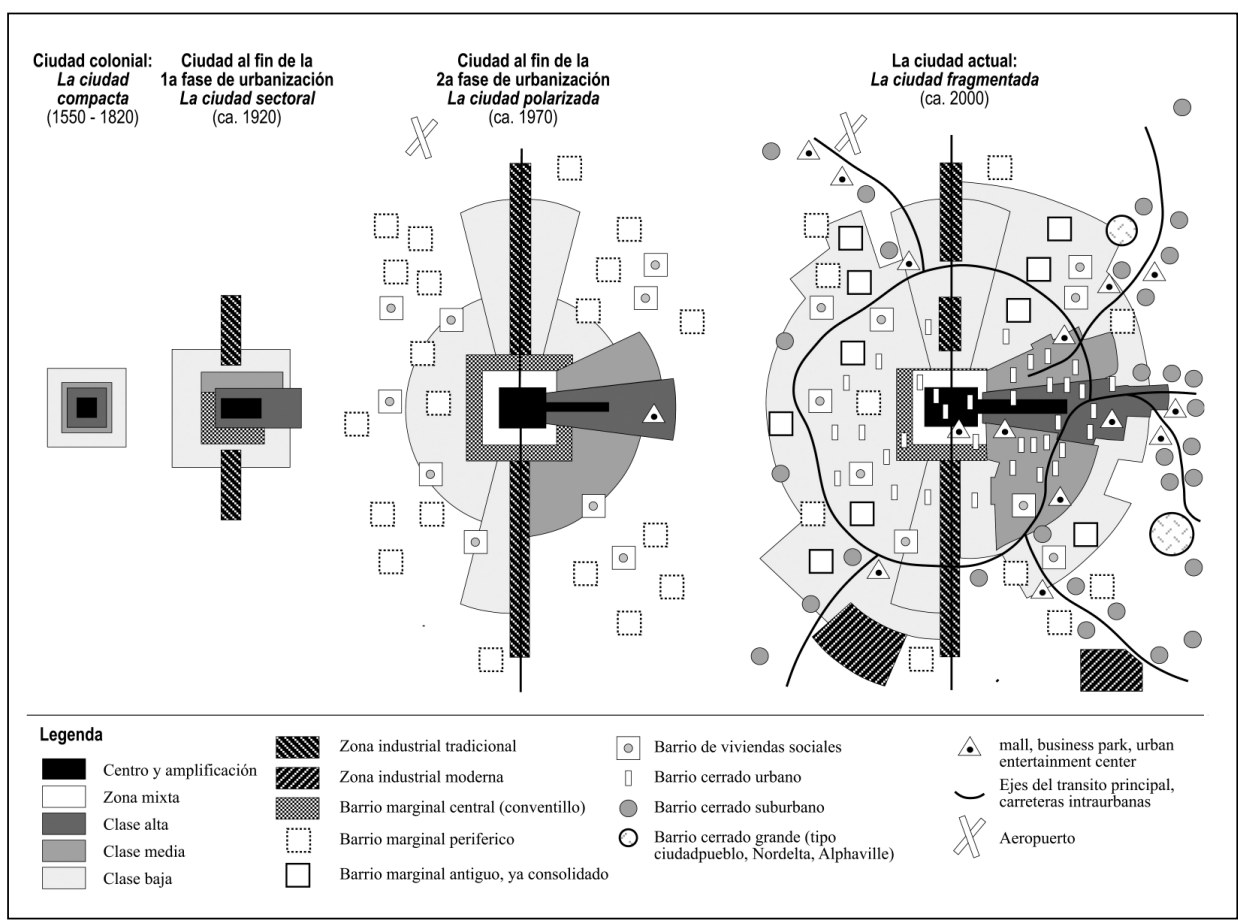

FIGURA 3. El modelo de desarrollo estructural de la ciudad latinoamericana 
En este última fase, debido a la influencia de la globalización, se han desarrollado cambios dramáticos en la estructura de las ciudades y en el desarrollo urbano (Borsdorf \& Hidalgo, 2005). Ello hizo necesario ampliar el modelo tradicional de desarrollo urbano de las ciudades latinoamericanas, estableciendo nuevas fases (Borsdorf, Bähr \& Janoschka, 2002; Borsdorf, 2003; Bähr $\&$ Borsdorf, 2005). Al igual que en Europa, también los nuevos modos de vencer las distancias (autopistas, sistema de metro y ampliación de los ferrocarriles metropolitanos) son una condición básica, además generan cambios en los modos de vida y nuevas demandas sobre el medio ambiente.

Bajo la influencia de estos factores, a pesar de la existencia de excepciones, se comprueba que las grandes ciudades allí, hoy crecen más lento que la población total; algunos autores hablan de un "fin del éxodo" en Latinoamérica (Borsdorf, 2004, Parnreiter, 2004). También en Latinoamérica el crecimiento urbano tuvo una nueva dimensión, en el sentido que las áreas periféricas de las grandes ciudades se volvieron más dinámicas que las áreas centrales de estas.

Sin embargo, los resultados son muy diferentes; si en muchos países de Europa occidental se puede observar que la creciente suburbanización de tendencias post-suburbanas será reemplazada, la evolución de estas tendencias en Latinoamérica todavía son muy difíciles de determinar. Estudios recientes muestran la existencia del fenómeno de "migración por amenidades", este es un ejemplo reciente observado en Santiago de Chile, donde la globalización se inició tempranamente y probablemente hasta hoy sus impactos han sido más investigados (Borsdorf \& Hidalgo, 2008).

Sin embargo, en general Latinoamérica se encuentra todavía en una fase de suburbanización (Escolano \& Ortiz, 2005). Los nuevos barrios residenciales, pero también los nuevos polígonos industriales y centros comerciales están todavía dentro de la frontera administrativa de la ciudad. Los nuevos barrios residenciales y "nuevas ciudades" que surgen en las "verdes praderas", fuera del espacio de urbanización cerrado, se relacionan estrechamente de manera funcional con el centro de la ciudad. Este fenómeno es en Europa similar, tanto en su forma como en el funcionamiento de la periurbanización (Hidalgo, 2005).

En la figura 4 se observa un modelo generalizado de la actual estructura fragmentada del Área Metropolitana de Santiago de Chile.

El proceso de globalización afecta en gran manera la disposición interna de las aglomeraciones. Se debe notar que la macrosegregación del espacio social de hace casi cien años se manifiesta en la expresión de una separación entre una "ciudad próspera" y una "ciudad pobre", es reemplazada hoy por la meso y micro segregación (Borsdorf \& Hidalgo, 2007). Esto se confirma con el desa- 


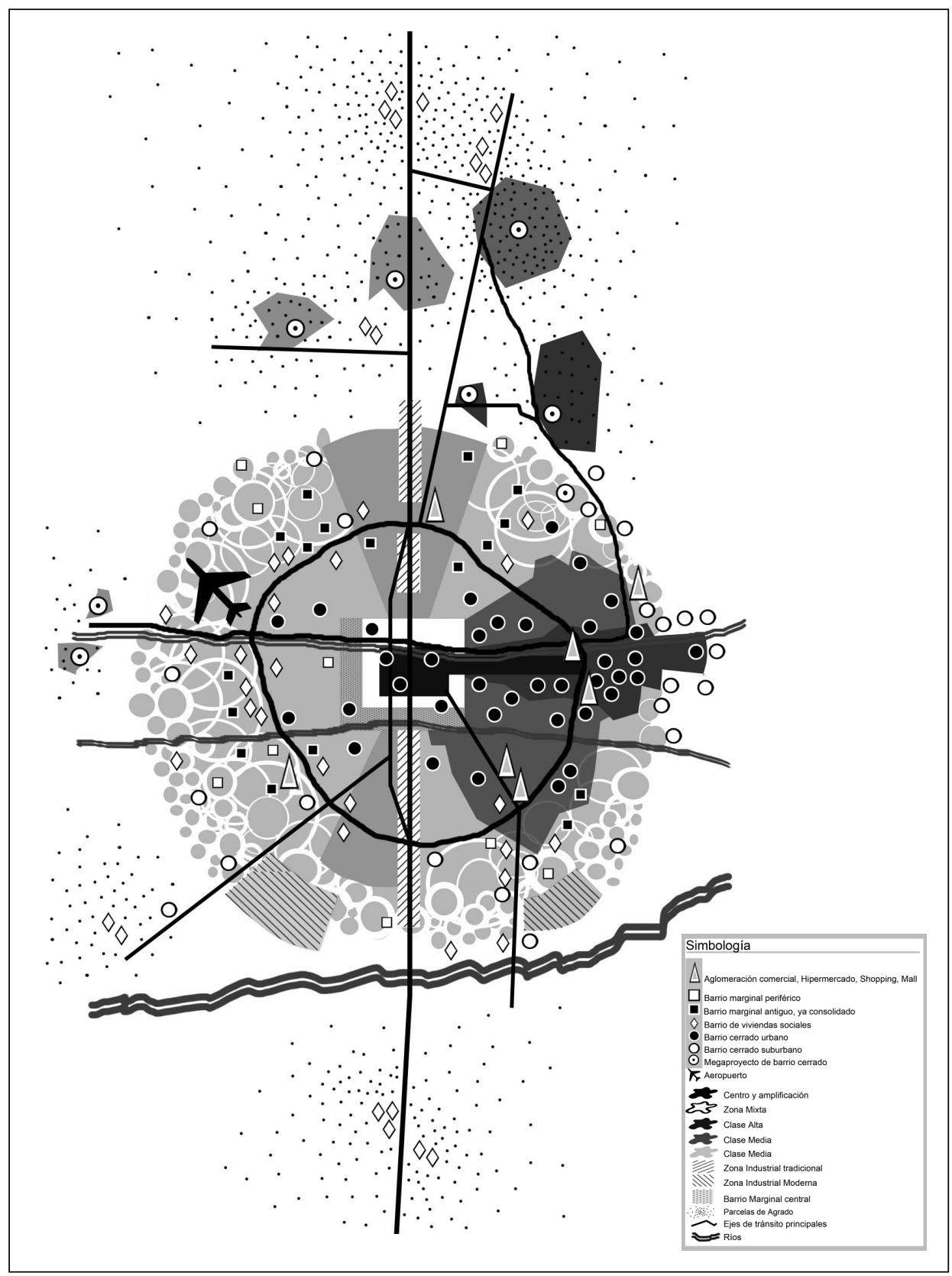

FIGURA 4. Modelo de estructura urbana de Santiago de Chile. (Elaboración propia) Estudios Geográficos, Vol. LXX, 266, pp. 181-203, enero-junio 2009 ISSN: 0014-1496, eISSN: 1988-8546, doi: 10.3989/estgeogr.0449 
rrollo de barrios cerrados, cuyas entradas son protegidas y vigiladas a toda hora. Estos barrios residenciales se constituyen como propiedad común (copropiedad), en ellas los propietarios adquieren una parte de la tierra y su vivienda. Dado que la propiedad privada puede cercarse, existe la posibilidad de cerrar grandes espacios residenciales, este es el caso de las "ciudades valladas" con más de 50.000 habitantes, grandes predios amurallados y espacios públicos privados. (Borsdorf \& Hidalgo, 2004; Hidalgo, Borsdorf \& Sánchez, 2007).

El prototipo de una ciudad vallada es Alphaville (São Paulo, Brasilien; Coy \& Pöhler, 2002), Nordelta en el Gran Buenos Aires (Janoschka, 2002, Janoschka \& Borsdorf, 2005), también Piedra Roja o Larapinta en las afueras de Santiago de Chile (Borsdorf \& Hidalgo, 2004) son ejemplos relevantes. La "condominización" (Plöger, 2006) se encuentra hoy también en los barrios tradicionales, donde las calles son cerradas convirtiéndose así en áreas privadas. Se puede hablar de meso y micro segregación, porque con el amurallamiento es posible crear barrios para clases medias y altas en entornos de menores recursos (Galleguillos, 2007). La proximidad entre ricos y pobres es vista por la población de mayores ingresos como positiva, ya que ellos necesitan personal para las labores domésticas y por lo tanto pueden reclutarlo fácilmente de sus alrededores.

Este proceso va de la mano con la creciente fragmentación del espacio urbano (Parnreiter, 2004) aparentemente sin planificación, surgen barrios residenciales por todos lados en la ciudad y forman, con sus torres en altura, visibles desde largas distancias, una imagen densa en forma de enjambre. También los Mall's y centros comerciales, que en los años setenta fueron motores del crecimiento urbano dirigido a la clase alta (Bähr \& Mertins, 1995), hoy se extienden por toda la ciudad, lo mismo ocurre con el establecimiento de universidades privadas, que sigue un modelo fractal.

En este contexto, Hidalgo (2007) ha examinado la difusión de los barrios de vivienda social en Santiago, concluyendo que hasta 1970 estos se encontraban en el interior de la aglomeración. Con el régimen militar (1973-1990) fueron saneadas las antiguas callampas (tugurios marginales) y los campamentos de emergencia, (autoconstrucciones de vivienda social) instalados bajo el gobierno de Allende, por otra parte, la población fue trasladada en gran parte a comunas tradicionales pobres dentro de la aglomeración (Galleguillos, 2007). Por ello en este período la vivienda social abandona las comunas ricas.

Solo con la llegada de la democracia y, particularmente, bajo los regimenes socialistas de Lagos y Bachelet fue esta política abandonada. En el llamado "barrio alto" no se construyeron nuevas viviendas sociales, esto se convirtió en un motor de la rápida suburbanización y de la expansión continua, cada 

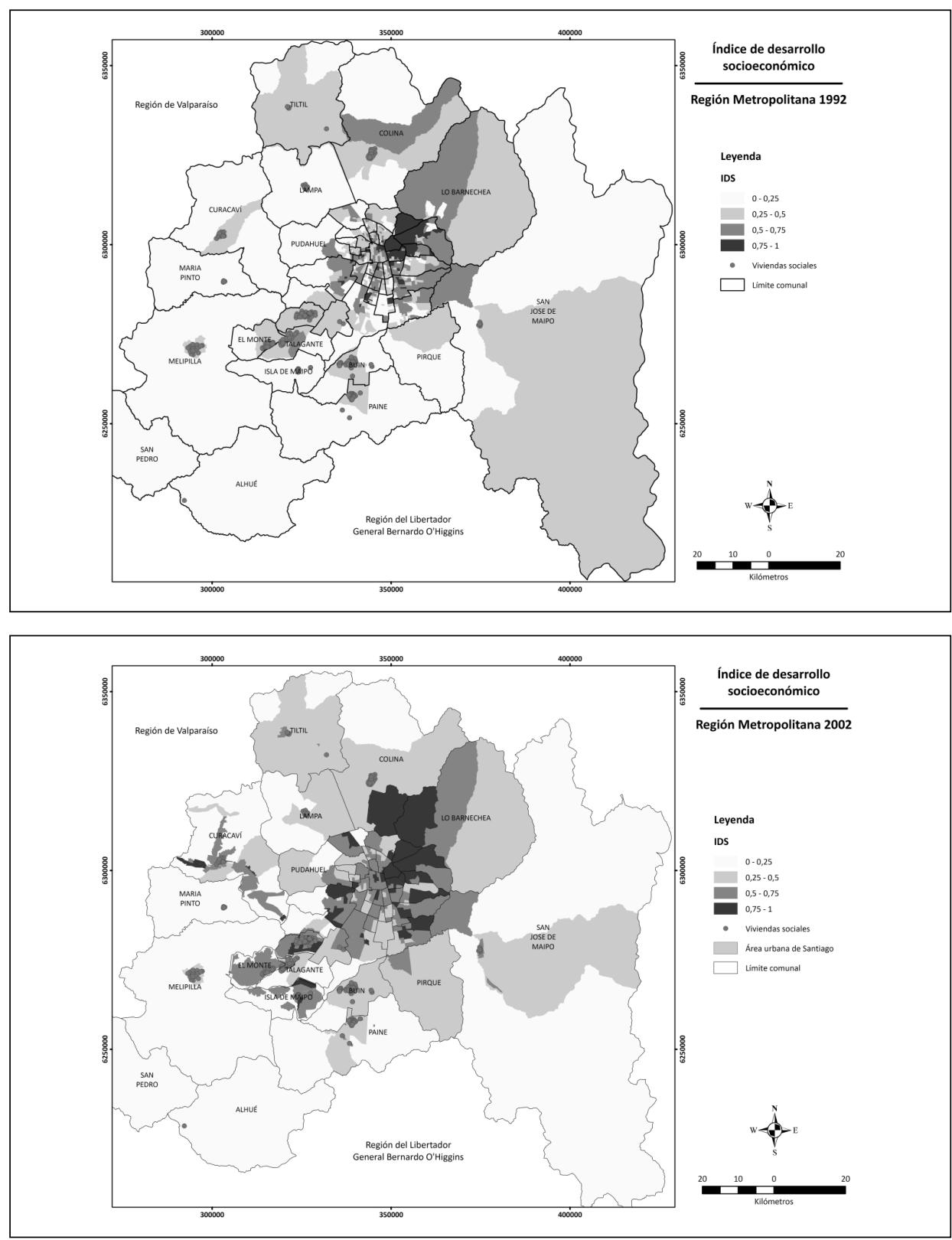

FIGURA 5. Distribución espacial de la construcción de vivienda social en Santiago de Chile 1992-2002 e Índice de Desarrollo Socioeconómico. Fuente: Hidalgo, 2008

Estudios Geográficos, Vol. LXX, 266, pp. 181-203, enero-junio 2009

ISSN: 0014-1496, eISSN: 1988-8546, doi: 10.3989/estgeogr.0449 
vez más alejada del centro de la ciudad y con construcciones en los prados verdes periféricos (figura 5 y tabla 3). La tabla número tres muestra que desde 1990 la construcción de vivienda social fuera del Área Metropolitana de Santiago fue dos veces mayor que en los años 1978-1989. Así el porcentaje de comunas periféricas aumenta constantemente (8,3\% de 1978-1983; 19,5\% en el período 1996-2002). Se observa también que la construcción de vivienda social bajo los gobiernos democráticos pierden importancia constantemente (ver al respecto Castillo \& Hidalgo, 2007).

TABLA 3

CONSTRUCCIÓN DE VIVIENDA SOCIAL DENTRO Y FUERA DEL ÁREA METROPOLITANA DE SANTIAGO DE CHILE 1978-2002

\begin{tabular}{lccccc}
\hline & $1978-1983$ & $1984-1989$ & $1990-1995$ & $1996-2002$ & Total \\
\hline $\begin{array}{l}\text { Construcción de vivienda social } \\
\text { fuera del Área Metropolitana } \\
\text { de Santiago, 1978-2003 (1) }\end{array}$ & 3.467 & 3.575 & 10.628 & 9.076 & 26.746 \\
\hline $\begin{array}{l}\text { Construcción de vivienda social } \\
\text { dentro del Área Metropolitana } \\
\text { de Santiago, 1978-2003 (2) }\end{array}$ & 37.908 & 68.804 & 55.268 & 37.369 & 199.349 \\
\hline \begin{tabular}{l} 
Total \\
\hline
\end{tabular} & 41.375 & 72.379 & 65.896 & 46.445 & 226.095 \\
\hline
\end{tabular}

(1) Comunas: Colina, Peñaflor, Padre Hurtado, Melipilla, Talagante, Buin, Paine, El Monte, Lampa, Isla de Maipú, Curacaví, Til-Til, San José de Maipú, María Pinto, Calera de Tango, Pirque, San José de Maipo y Alhué.

(2) Comunas: Puente Alto, La Pintana, San Bernardo, La Florida, Maipú, Pudahuel, Renca, El Bosque, Peñalolén, Quilicura, La Granja, Lo Prado, Macul, Cerrillos, Cerro Navia, Lo Barnechea, San Ramón, Conchalí, Lo Espejo, Estación Central, Las Condes, Pedro Aguirre Cerda, Huechuraba, Santiago, La Reina, San Joaquín, La Cisterna, Ñuñoa, Providencia, Recoleta, San Miguel y Quinta Normal.

Fuente: Hidalgo, 2008.

En el transcurso de este desarrollo aumenta la migración diaria (migración pendular) en la aglomeración. La figura 6 muestra que esto todavía se desarrolla de acuerdo al patrón suburbano.

Para la clase alta en los últimos años, ha crecido la posibilidad de adquirir una segunda residencia, pero en algunos casos también una residencia principal a gran distancia de la aglomeración. Este nueva ciudad no escapa a las estructuras post-suburbanas, este es mas bien un fenómeno de migración por 


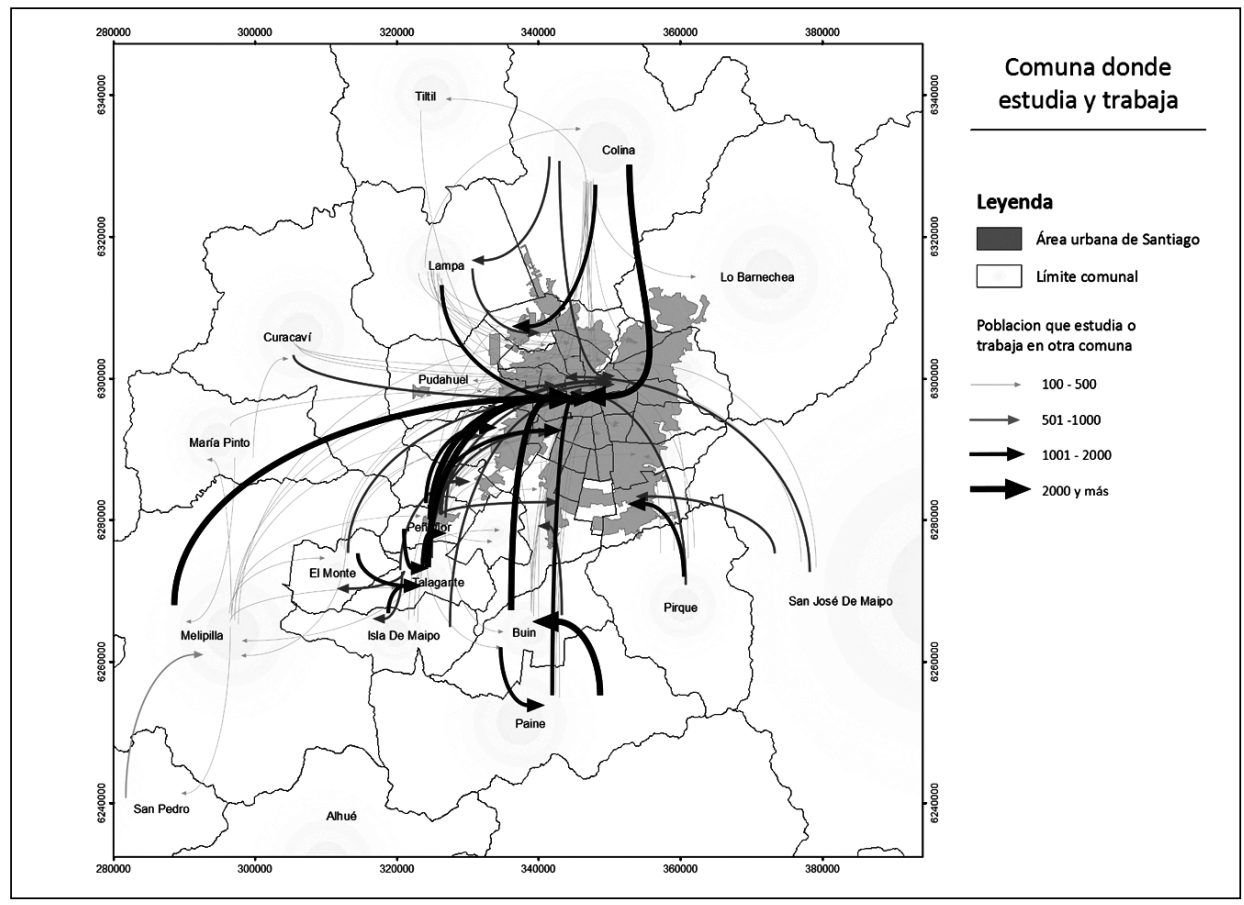

Fuente: Hidalgo, 2008.

FIgURA 6. Migración diaria en el Área Metropolitana de Santiago de Chile 2002

amenidades y por consiguiente, de desurbanización, como se conoce muy bien en los Estados Unidos. En Santiago este fenómeno incluye la Cordillera de la Costa y el Cajón del Maipo, un valle en lo alto de la Cordillera de los Andes, donde existen grandes terrenos con más de $5.000 \mathrm{~m}^{2}$ llamadas "parcelas de agrado", estas son viviendas diseñadas de manera individual que poseen equipamientos tales como piscinas (figura 7). La Cordillera de la Costa es un nuevo lugar atractivo para residencia de las clases altas de la segunda Área Metropolitana más grande del país (Valparaíso-Viña del Mar) (Borsdorf \& Hidalgo, 2008b). También las parcelas de agrado se encuentran en gran medida en complejos similares a los condominios cerrados, con vigilancia durante todo el día.

El pueblo vacacional de Olmué, ubicado a 80 kilómetros de la capital es un ejemplo de este tipo de desarrollo (figura 7). Factores de atracción son la buena calidad del aire, particularmente saludable por la existencia de un mi- 


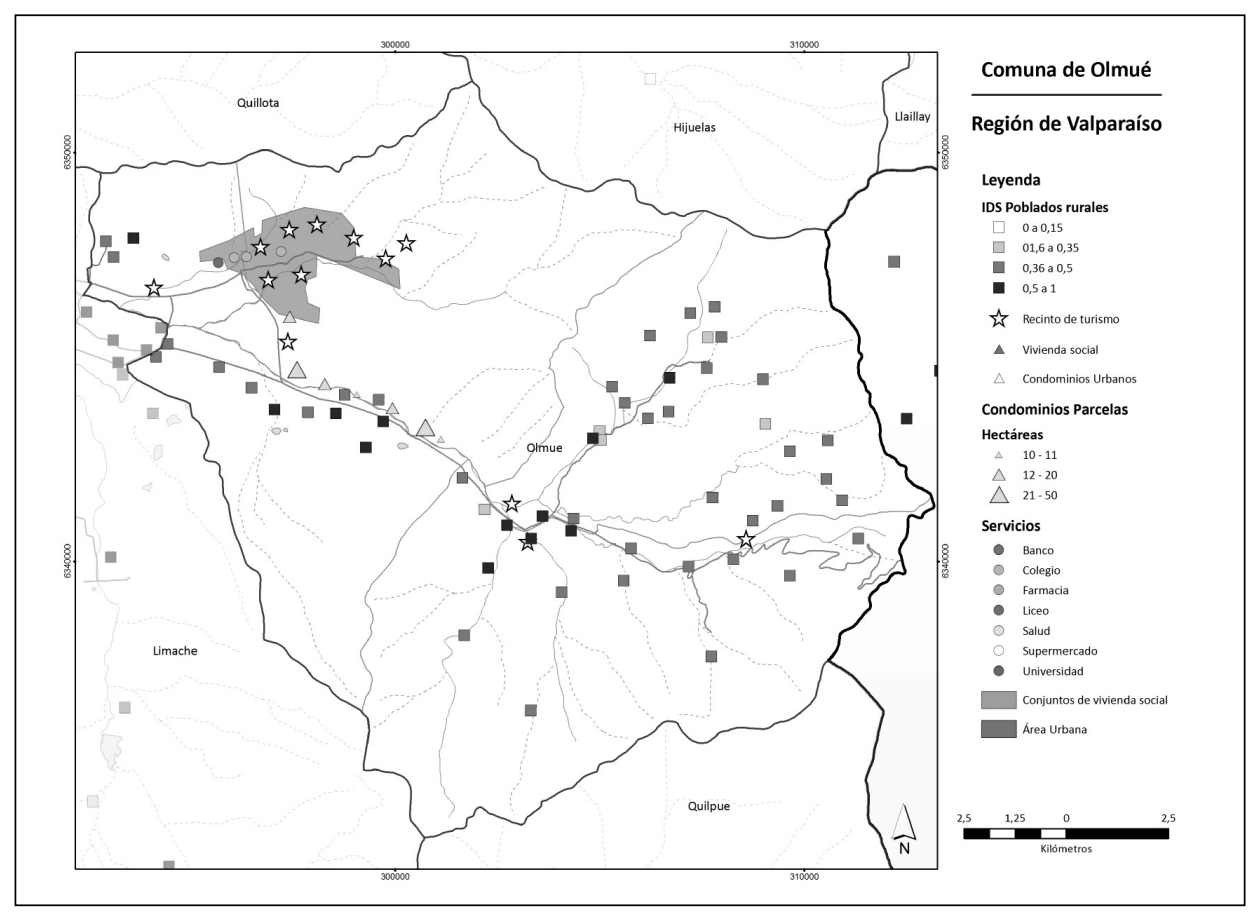

Fuente: Elaboración propia.

FIgURA 7. Condominios y parcelas de migrantes por amenidades en la comuna de Olmué, Chile e indice de Desarrollo Socioeconómico de los poblados rurales en la comuna

croclima, la belleza del paisaje, la cercanía de un parque nacional y reserva de la biósfera, la cultura rural materializada en el rodeo y otras "amenidades". Este desarrollo fue posible en primer lugar con la pavimentación de las vías de acceso, que reduce el tiempo de viaje de dos a una hora desde Santiago y la existencia de una flota de buses permanente de buena calidad; el precio de un viaje ida y vuelta es menos de 10 euros, esto para los chilenos de ingresos medios no es ahora un impedimento y se encuentra dentro de los costos que, hasta la implementación del sistema de tarifas unificadas (2007) para los barrios periféricos, se pueden emplear también dentro de la aglomeración.

Las "amenidades" del valle cordillerano Cajón del Maipo son todavía más atractivas que las de Olmué. La cordillera es alta, existen centros de esquí en los alrededores, el río permite el desarrollo de rafting, la infraestructura turística, también para los migrantes de fin de semana, es antigua y por ello "au- 
téntica", la cultura, similar a los valles alpinos, es autóctona. Los parques naturales invitan a caminar, a disfrutar los manantiales. Pero más aún, la distancia al centro de la ciudad es solo alrededor de 50 kilómetros y el tiempo de viaje inferior a una hora, como en Olmué.

La localización de residencias en zonas periféricas extremas no se debe solo a la existencia de "amenidades". El ejemplo de Ciudad de México demuestra que el deseo de exclusividad es un elemento importante en la formación de las preferencias residenciales. Quien reside en el Estado de México, escoge por ejemplo su residencia en villas exclusivas o en condominios suburbanos como Santa Fe; la distancia al trabajo se soluciona con el uso de cómodos helicópteros, dada la existencia de un cierto número de edificios que cuentan con helipuertos (Kohler, 2005).

Los ejemplos muestran que Latinoamérica no ha estado ausente a los procesos experimentados en los Estados Unidos como la Exurbanización y las Edge Cities. Sin embargo, si bien pueden existir tendencias de post-suburbanización, como por ejemplo que las principales sedes de empresas internacionales, se presentan bastante móviles y en forma fragmentada, todavía no se han atrevido a traspasar los límites de las fronteras administrativas de la metrópolis.

\section{CONCLUSIÓN}

Alrededor del mundo las estructuras urbanas se encuentran en transición. Esto es válido para el caso de Europa y Latinoamérica; una fuerza motora de ello es la globalización. Sin embargo, ello no conduce (todavía) a la homogenización. Al menos en Europa continental occidental, desarrollos post-suburbanos como los percibidos en Latinoamérica son todavía desconocidos; allí las tradicionales tendencias de segregación social, macro, meso y microsegregación han sido reemplazadas. El crecimiento urbano todavía ocurre en la forma de suburbanización, donde la vivienda social es un elemento clave del desarrollo de la ciudad. En una primera aproximación es percibido el fenómeno de "migración por amenidades", este es posible de encontrar también en otros espacios culturales del planeta (Moss 2006) e incluso es conocido en Europa (Perlik 2006). Estas formas de "evitar la ciudad" son por tanto, una forma de desurbanización. Ellas son responsables de que hoy las grandes aglomeraciones latinoamericanas crezcan menos rápido que la población total. Algunas ciudades latinoamericanas han iniciado el desarrollo de las primeras medidas de renovación urbana fuera de los centros urbanos, con el fin de establecer un 
cambio de tendencia hacia una reurbanización. Habrá que esperar para saber si éstas medidas son exitosas.

Recibido: 02/03/2009

Aceptado: 30/03/2009

\section{BiBLIOGRAFÍA}

Bähr, J., Borsdorf, A. (2005): La ciudad latinoamericana. La construcción de un modelo. Vigencia y perspectivas. In: Urbe. Revista de ciudad, urbanismo y paisaje (Lima), 2, 2, S. 207-222.

Bähr, J., Mertins, G. (1995): Die lateinamerikanische Stadt. Erträge der Forschung. Darmstadt.

Borsdorf, A., (2002): Barrios cerrados en Santiago de Chile, Quito y Lima: tendencias de la segregación socio-espacial en capitales andinas. In: Cabrales Barajas, L.F. (ed.) Latinoamérica: países abiertos, ciudades cerradas. Guadalajara: UNESCO, S. 581-610.

Borsdorf, A. (2002a): Urbane Transformation in Lateinamerika: Von der polarisierten zur fragmentierten Stadt. In: GW-Unterricht 89, S. 23-33.

Borsdorf, A. (2002b): Innsbruck-from City to Cyta? Outskirt development as an indicator of spatial, economic and social development. In: Dubois-Taine, G. (Hrsg.) From Helsinki to Nicosia. Eleven Case Studies \& Synthesis. Paris, S. 57-74.

Borsdorf, A., (2003): Cómo modelar el desarrollo y la dinámica de la ciudad latinoamericana. EURE Revista Latinoamericana de Estudios Urbano Regionales 29, 86, S. $37-49$.

Borsdorf, A., (2004): On the way to post-suburbia? Changing structures in the outskirts of Euopean cities. In: Borsdorf, A. \& P. Zembri (Hg.): Structures. European Cities: Insights on Outskirts. Paris, S. 7-30.

Borsdorf, A. (2004a): Landflucht als Teil der Mobilitätstransformation. Das Beispiel Lateinamerika. In: Praxis Geographie 34, 7-8, S. 9-14.

Borsdorf, A. (2004b): Wenn Stadt "geformter Geist" ist, wofür steht dann Postsuburbia? Spurenlesen im rurbanen Raum. In: Borsdorf, A. \& V. Mayer (Hg.), 2004: Konvergenz und Divergenz der Kulturen in den Randzonen der Städte. Beiträge der Sektion 3.7. Internationale Konferenz Das Verbindende der Kulturen. Schriftenreihe Wohnwesen Umland Wien 2, Wien, S. 17-25.

Borsdorf, A. (2005): Introduction: Évolutions postsuburbaines en Europe et dans le Nouveau Monde. Revue de Géographie de l'Est 45, 3-4, S. 125-132.

Borsdorf, A., Bähr, J. and Janoschka, M. (2002): Die Dynamik stadtstrukturellen Wandels in Lateinamerika im Modell der lateinamerikanischen Stadt. Geographica Helvetica $57 / 4$, S. 300-310 
Borsdorf, A. and R. Hidalgo (2004): Vom Barrio Cerrado zur Ciudad Vallada. Neue Dimensionen der sozialen und funktionalen Exklusion in Santiago de Chile. Mitteilungen der Österreichischen Geographischen Gesellschaft 146, S. 111124.

Borsdorf, A. and R. Hidalgo, (2005): Städtebauliche Megaprojekte im Umland lateinamerikanischer Metropolen-eine Antithese zur Stadt? Geographische Rundschau 57, 10, S. 30-39.

Borsdorf, A. and R. Hidalgo (2007): A new model of urban development in Latin America: The Gated Communities and Fenced Cities in Santiago de Chile and Valparaíso. Cities. The International Journal of Urban Policy and Planning 24, 5, S. 365-378.

Borsdorf, A. and Hidalgo, R. (2008): New dimensions of social exclusion in Latinamerica: From gated communities to gated cities. The example of Santiago de Chile. Land Use Policy 25, 2, S. 153-160.

Borsdorf, A. and Hidalgo, R. (2008a): Offener Hafen-geschlossene Wohnviertel? Urbane Transformation in der Struktur der Metropolitanregion von Valparaíso, Chile. In: Erdkunde (im Druck).

Borsdorf, A. and Hidalgo, R. (2008b): Pleasure Lots near Santiago de Chile and Valparaíso. The Chilean way of Amenity Migration. In: Moss, L. (Hrsg.): Proceedings of the Amenity Migration Conference at Banff/Canada 2008. (in Vorb.)

Borsdorf, A. and P. Zembri (Hrsg.) (2004): Structures. European Cities: Insights on Outskirts. Paris.

Borsdorf, A., et al. (2004): Parallels and differences in the outskirts of European cities. A. methodological reflection and a comparative matrix. In: Borsdorf, A. and P. Zembri (Hg.), 2004: Structures. European Cities: Insights on Outskirts. Paris, S. 169-181.

Brake, K., Dangschat J. S. and Herfert, G. (Hrsg.) (2001): Suburbanisierung in Deutschland. Aktuelle Tendenzen. Opladen: Leske + Budrich.

Burdack, J. (2001): Die städtische Peripherie zwischen "suburbanen" und "postsuburbanen" Entwicklungen. In: Berichte zur deutschen Landeskunde 75, 2-3, S.188-196.

Burdack, J., Herfert, G. and Rudolph, R. (Hrsg.) (2005): Europäische metropolitane Peripherien. Beiträge zur Regionalen Geographie 61. Leipzig: Institut für Länderkunde.

Castillo, M. J. and Hidalgo, R. (Hrsg.) (2007), 1906-2006. Cien años de política de vivienda en Chile. Santiago de Chile: Alvimpress.

Coy, M. and Pöhler, M. (2002): Condomínios fechados und die Fragmentierung der brasilianischen Stadt. Typen-Akteure-Folgewirkungen. In: Geographica Helvetica, 57,4 , S. 264-277.

Dubois-Taine, G. (2004a): Outskirts of European cities. Understand better, govern better. In: Dubois-Taine, G. (Hrsg.): From Helsinki to Nicosia. Eleven case studies \& synthesis. Paris, S. 7-55. 
Dubois-Taine, G. (2004b): Biarritz-Anglet-Bayonne-San Sebastian. How to organize and territory town? In: Dubois-Taine, G. (Hrsg.) From Helsinki to Nicosia. Eleven Case Studies \& Synthesis. Paris, S. 57-76.

Escolano, S. and Ortiz, J. (2005): Processus de fragmentation des structures spatiales du Grand Santiago (Chili). In: Revue Géographique de l'Est 3-4, 195-208.

Eisinger, A. and Schneider, M. (Hrsg.) (2003): Stadtland Schweiz. Untersuchungen und Fallstudien zur räumlichen Struktur und Entwicklung in der Schweiz. Basel, Boston und Berlin.

Galleguillos Araya-Schübelin, M. X. (2007): Möglichkeiten zum Abbau von Segregation in Armenvierteln. Die Frage nach der sozialen und ökonomischen Nachhaltigkeit urbaner Ballungsräume am Beispiel Santiago de Chile. Kieler Geographische Schriften 115. Kiel.

Garreau, J. (1991): Edge City: Life on the New Frontier. New York: Doubleday.

Hidalgo, R. (2005): Post-suburbia o post-urbia? Les mégaprojets résidentiels dans la périphérie de Santiago du Chili. Revue Géographique de l'Est 3-4, 209-218.

Hidalgo, R. (2007): Cien años de política de vivienda social, cien años de expulsión de los pobres a la periferia de Santiago. In: Castillo, M. J., Hidalgo, R. (Hrsg.) (2007): 1906-2006. Cien años de política de vivienda en Chile. Santiago de Chile, S. 51-63.

Hidalgo, R. and A. Borsdorf (2005): La exclusion residencial y el desarrollo de la ciudad moderna en América Latina: De la polarización a la fragmentación. El caso de Santiago de Chile. Geographicalia (Zaragoza) 48, S. 5-29.

Hidalgo, R., Borsdorf, A. and Sánchez, R. (2007): Hacia un nuevo tejido rurbano. Los megaproyectos de ciudades valladas en la periferia de Santiago de Chile. Ciudad y Territorio. Estudios Territoriales (Madrid) 39, 151, S. 115-135.

Janoschka, M. (2002): Wohlstand hinter Mauern. ISR Forschungsberichte 28, Wien: ÖAW.

Janoschka, M. and A. Borsdorf (2005): Condominios fechados. The rise of private residential neighbourhoods in Latin America. In: Glasze, G., Webster, C. and Frantz, K. (eds.): Private Cities. Global and Local Perspectives. London \& New York: Routledge, S. 92-108.

Kanitscheider, S. (2002): Condominios und fraccionamientos cerrados in MexikoStadt. Sozialräumliche Segregation am Beispiel abgesperrter Wohnviertel. In: Geographica Helvetica 57 (4), S. 253-263.

Kohler, P. (2002): Geschlossene Wohnviertel in Quito. Naturraum und rechtliche Rahmenbedingungen als Einflussgrößen für Verbreitung und Typisierung. In: Geographica Helvetica 57 (4), S. 278-289.

Kohler, P. (2005): Ökonomische Transformation und Fragmentierung der urbanen Struktur von Mexiko Stadt. Zur Maßstäblichkeit postmoderner Stadtentwicklungsprozesse. Dissertation. Innsbruck.

Longchamp, J. (1989): La périurbanisation dans l'ouest lémanique. Lausanne.

Meyer, K. and J. Bähr (2001): Condominios in Greater Santiago de Chile and their Impact on the Urban Structure. Die Erde 132, 3, S. 293-321 
Moss, L. (2006): The Amenity Migrants. Seeking and sustaining mountains and their cultures. Oxford and Cambridge (Mass.)

Parnreiter, C. (2004): Zwischen hoher Verstädterung und neuer ländlicher Entwurzelung. Migration in Lateinamerika. In: Praxis Geographie 7-8, 34-37.

Parnreiter, C. (2004): Entwicklungstendenzen lateinamerikanischer Metropolen im Zeitalter der Globalisierung. In: Mitteilungen der Österreichischen Geographischen Gesellschaft, 146. S. 1-28.

Parnreiter, C. (2005): Historische Geographien, verräumlichte Geschichte: Die lateinamerikanische Stadt im 20. Jahrhundert. Habilitationsschrift Wien.

Perlik, M. (2006): The Specifics of Amenity Migration in the European Alps. In: MOSS, L.A.G.: The Amenity Migrants: Seeking and Sustaining Mountains and Their Cultures. Wallingford/UK, Cambridge, USA: CABI, S. 215-231.

Piron, O., Dubois-Taine, G. (1998): La ville émergente. Constats pour renouveler les lignes d'actions publiques. Paris.

Plöger, J. (2006), Die nachträglich abgeschotteten Nachbarschaften in Lima (Peru). Eine Analyse sozialräumlicher Kontrollmaßnahmen im Kontext zunehmender Unsicherheiten. Kieler Geographische Schriften 112. Kiel.

Schneider-Sliwa, R. (Hrsg.) (2002): Städte im Umbruch. Berlin: Reimer.

Sieverts, T. (1997): Zwischenstadt. Zwischen Ort und Welt, Raum und Zeit, Stadt und Land. Bauwelt Fundamente 113. Braunschweig und Wiesbaden.

Watson, S. and Gibson, K. (Hrsg.) (1995): Postmodern Cities and Spaces. Oxford.

Wood, G. (2003): Die postmoderne Stadt. Neue Formen der Urbanität im Übergang vom zweiten ins dritte Jahrtausend. In: Gebhardt, H., Reuber, P., Wolkersdorfer, G. (Hrsg.): Kulturgeographie. Aktuelle Ansätze und Entwicklungen. Heidelberg, Berlin: Spektrum Akademischer Verlag, S. 131-147.

Wilhelmy, H. (1952): Südamerika im Spiegel seiner Städte. Hamburg.

Wilhelmy, H. and Borsdorf, A. (1984, 1985): Die Städte Südamerikas. 2 Bände. Berlin und Stuttgart: Bornträger.

\section{RESUMEN}

A partir del desarrollo urbano en Europa se hacen algunas consideraciones conceptuales de los principales procesos que han marcado a la morfología y estructura de las ciudades. A partir de estas disquisiciones se interpreta de un modo general la evolución de la ciudad latinomamericana hasta los tiempos modernos. En base a la transformaciones ocurridas en Santiago de Chile en las últimas décadas se intenta comparar y poner en tensión el marco teórico generado para la realidad europea.

PALABRAS ClAVE: transformaciones metropolitanas; modelos de desarrollo urbano; policentralidad; postsuburbio; migración por amenidades. 


\begin{abstract}
Based on the urban development in Europe, this paper includes conceptual deliberations on the main processes which have affected both the morphology and structure of cities and it also interprets in broad terms the evolution of the Latin American city up to the modern times. On the basis of the transformations occurred in Santiago of Chile over the last decades, this study aims to draw a comparison with the theoretical framework set for the European reality.

KEY WORDS: metropolitan transformations; models of urban development; policentrality; postsuburb; amenity migration.

\section{RÉSUMÉ}

À partir du développement urbain en Europe, cet article inclut quelques réflexions conceptuelles sur les principaux processus qui ont eu une influence sur la morphologie et la structure des villes. À partir de ces analyses, on interprète en général l'évolution de la ville latino-américaine jusqu'à l'époque moderne. Sur la base des transformations qui ont eu lieu à Santiago du Chili au cours des dernières décennies, on envisage de faire la comparaison avec le cadre théorique créé pour la réalité européenne.
\end{abstract}

MoTS CLÉs: transformations métropolitaines; modèles de développement urbain; policentralité; post-banlieue; migration d'agrément. 